46

...select older
persons may
be excellent
candidates
for kidney
donation...

\title{
New tool for prediction of ESRD risk in living kidney donor candidates
}

Current evaluation of candidates for living kidney donation involves screening for individual risk factors for end-stage renal disease (ESRD). Now, researchers have developed an online tool to aid donor selection that predicts the long-term risk of ESRD in donor candidates in the absence of kidney donation.

"Although prediction models to estimate ESRD risk in patients with chronic kidney disease exist, there were no models for low-risk individuals who would be considered suitable candidates for kidney donation," explains researcher Morgan Grams. "Developing this type of model is difficult because very few ESRD events occur in lowrisk individuals, and few cohorts of low-risk individuals have been followed for ESRD."

To develop their risk prediction tool, Grams and colleagues identified nearly 5 million individuals with no health conditions that

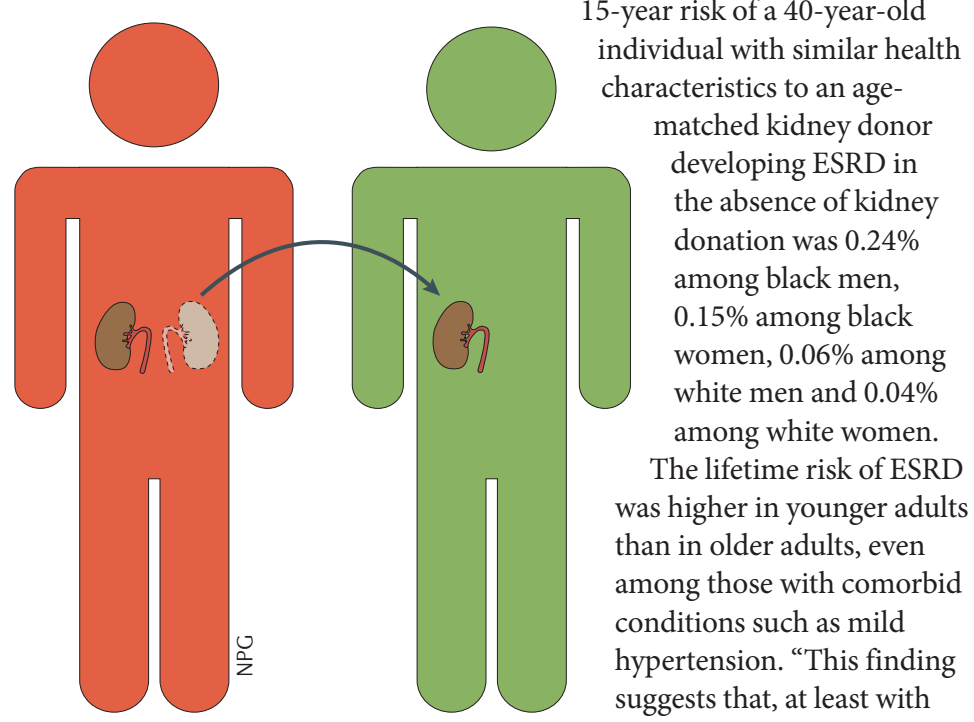

would be considered absolute contraindications for kidney donation, from seven general population cohorts in the USA, Canada and Israel. They assessed the relative risks of ESRD associated with ten demographic and health characteristics in these low-risk subgroups, which had follow-up data ranging from a median of 4 years to 16 years, and calibrated these risk associations to the observed incidence of ESRD among low-risk individuals in the USA. "We used this method because relative risks tend to be fairly generalizable, whereas absolute risks can vary from population to population. Thus, we wanted to anchor our absolute risks on the most generalizable population we could," says Grams.

The researchers found that the long-term risk of ESRD among lowrisk individuals varies considerably according to age, sex and race.

According to their model, the 15-year risk of a 40-year-old individual with similar health aracteristics to an agematched kidney donor developing ESRD in the absence of kidney donation was $0.24 \%$ among black men, $0.15 \%$ among black women, $0.06 \%$ among white men and $0.04 \%$ among white women.

The lifetime risk of ESRD was higher in younger adults han in older adults, even among those with comorbid hypertension. "This finding suggests that, at least with respect to their future risk of ESRD, select older persons may be excellent candidates for kidney donation," comments Grams. Consistent with previous estimates of the relative risk associated with kidney donation, the observed risk of ESRD in US donors 15 years after donation was 3.5-5.3 times higher than the projected risk in the absence of donation.

"Our model is included in the draft KDIGO clinical practice guidelines for the evaluation and follow-up care of living kidney donors, which were recently sent out for public review and comment," says Grams. She suggests that projected risks of ESRD could be used by transplant centres to determine which donor candidates should be accepted for donation, as well as to counsel candidates with respect to their long-term risk of kidney disease. The researchers plan to update their risk model by incorporating new data when it becomes available.

"It is important to note that our risk tool estimates the predonation risk-that is, the longterm risk of ESRD if the donor candidate does not donate a kidney," says Grams. "At the present time, we do not have sufficient data to estimate the post-donation risk of donor candidates, and to determine whether this risk varies by predonation demographic and health characteristics, but we hope to address these areas in the future."

Ellen F. Carney 